\title{
Stress and Violence in Video Games: Their Influence on Aggression
}

\author{
Genicelle Barrington $^{1}$ - Christopher J. Ferguson ${ }^{1}$
}

Accepted: 4 January 2022 / Published online: 21 January 2022

(c) Associação Brasileira de Psicologia 2022

\begin{abstract}
This study investigated whether stress or violent content in video games plays a greater role in aggressiveness towards a cooperative partner while playing a video game. It was hypothesized that participants, when exposed to stress, would demonstrate greater aggressiveness toward an incompetent partner than a competent partner. Furthermore, it was hypothesized that participants, when exposed to a violent video game, would demonstrate greater aggression toward an incompetent partner than those exposed to a non-violent video game. Stress was provoked in half of the participants using the Paced Auditory Serial Addition Test (PASAT), while others took a simple math quiz. Participants were then assigned to a video game condition, violent or non-violent with a competent or incompetent confederate and completed a reaction time task to measure aggression. Results indicated that provoked stress and violent content are not linked to aggression in this context.
\end{abstract}

Keywords Stress · Aggressiveness · Video games · Violent

Under stressful circumstances, individuals tend to resort to aggressiveness. Studies have highlighted that stressful situations which have developed earlier in life and are repeatedly imposed on an individual have underlying effects on aggression. However, many studies have also discussed the perception that immediate stress can also provoke and incite aggression (Verona \& Kilmer, 2007). Despite this, it is important to understand whether acquired stress plays a role in the expression of human aggression.

Genicelle Barrington

gbarrington@stetson.edu

Christopher J. Ferguson

CJFerguson1111@aol.com

1 Department of Psychology, Stetson University, Campus Box 6048, Deland, FL 32723, USA 


\section{Animals, Stress, and Aggression}

One means of examining how stress impacts aggression is through the study of animal models. In one study conducted with male adult mice, acute stressors were the primary causes of a marked increase in aggressiveness (Nosjean et al., 2015). During this study, the researchers' aim was to determine the immediate effect acute stress would have on social interaction in adult male mice. In order to conduct their study, the researchers used 70 adult male mice purchased from a laboratory and separated them into isolated host $(\mathrm{IH})$ mice and social visitor (SV) mice. During the study, each isolated host mice either received or did not receive acute stress while the social visitor mice remained unstressed throughout the environment. After being placed under duress, the IH mice were allowed to explore their cage before being exposed to an SV mouse for social interaction. This interaction was videotaped and analyzed by researchers. The researchers concluded that stress depleted the mice's social repertoire, accelerated their dominance behaviors, and furthered aggressive behaviors. Due to their findings, they concluded that social interactions can be influenced by a single stressful event.

Kohl et al. (2013) utilized a similar procedure, wherein mice were exposed to a resident intruder to measure and evaluate the resident's aggressive behaviors. In their study, they primarily focused on whether there was an interaction of conditional neural cell adhesion molecule knockout (NCAM-KO) and the extent to which exposure to repeated stress influenced aggression. Through the utilization of a gene by environment experiment, researchers attempted to determine whether the lack of forebrain in NCAM-KO mice would facilitate the development of an aggressive phenotype following exposure to either subchronic stress and chronic stress or one stressful condition when compared to wild-type variations of the mice. During their procedure, the researchers first measured the animals' baseline anxiety-like behavior through the use of an elevated plusmaze; they also conducted a bedding preference test to determine social behavior, an open field test to observe exploratory behavior, and the resident-intruder test for aggression. After running statistical analyses on their data, they found that NCAM-KO mice exhibited no differences in aggressive behavior prior to subchronic stress exposure and while under basal conditions. However, following chronic exposure to stress, the NCAM-KO mice showed increased aggressive behavior toward their intruder. These findings led to the conclusion that chronic stress-induced differences in aggressive behavior rather than subchronic stress. Furthermore, the researchers outlined that due to the age of their mice, there could be age differences in the vulnerability to stress and thus a differing impact of stress on aggression.

Studies such as these provide some useful hints as to how stress can influence animals. However, mice are not humans, and it is to human research that we next turn.

\section{Humans, Stress, and Aggression}

It has been long suggested as part of the Frustration-Aggression Hypothesis that certain types of stress may provoke aggression. Other scholars have suggested that stress more generally, not merely frustration, could lead to aggression. Hinsberger 
et al. (2016) aimed to determine whether individuals were more aggressive when exposed to continuous traumatic stress. Researchers had trained mental health experts and counselors to conduct diagnostic interviews of 290 South African participants. The researchers discovered that there was a significant correlation between self and witnessed traumatic events, PTSD symptom severity, and engagement in violent behavior. Researchers found that attraction to violence was predicted by witnessed traumatic events as well as victimization, and perpetrated acts of violence were directly predicted by the witnessing of violence and that PTSD symptom severity was directly predicted by victimization. They also found that exposure to violence did not directly influence PTSD severity or perpetrated violence by means of appetitive aggression. Instead, they determined that appetitive aggression predicted the severity of PTSD and aggressive behavior. This study primarily focused on the act of resorting to violence when under continually stressful circumstances and concluded that aggression exhibited in stressful environments would aid in survival.

In a study conducted by Verona and Kilmer (2007), acute stress was examined as a determinant of aggressive behaviors in 120 volunteers. The researchers used the Positive and Negative Affect Schedule before and after a stress manipulation. Participants were exposed to either low- and high-stress conditions after which they interacted with a confederate who they could inflict with shocks during judgment task. In their study, the researchers primarily found gender differences in the influence of acute stressors on aggressive behaviors. They concluded that women displayed less aggression whereas men displayed increased aggression after being exposed to the high-stress condition.

Meta-analyses likewise have examined the relationship between stress and aggression. Evidence suggests that stress and trauma can increase the propensity to engage in aggression in both men and women (Augsburger, \& Maercker, 2020; Orth, $\&$ Wieland, 2006). Such effects appear fairly consistent in the literature with moderate to large effects for hostile feelings and small to moderate effects for aggressive behavior.

\section{Violence in Video Games}

Prior studies outlined observed aggression in relation to stress; however, in this study, it is important to understand whether the task the participant engaged in also influences aggression, especially under stressful circumstances. Therefore, it is important to also understand the relationship between video games and aggression. The issue of whether violence in video games can impact aggression has been controversial. Despite several decades of research, no consensus has emerged. Though the American Psychological Association (APA) has taken a stance that violent games promote aggression (but not violent crime), reanalysis of their work has suggested that this claim is not supported by the extant literature and the APA may be misinforming the public (Ferguson et al., 2020). Below, we consider just a few studies in this realm.

In a unique study designed by Weber et al. (2006), researchers analyzed brain activity in relation to game play to understand and distinguish between virtual 
violence and non-virtual violence. The researchers conducted their experiment using 13 volunteers who chose to play a mature-rated first-person shooter game while under fMRI scanning. They recorded brain activity, physiological responses throughout game play, audio data from the game, and video display of the game play. They also included a questionnaire measure meant to control for arousal and subjective experiences throughout the experiment. After analyzing their data, they found that virtual violence produces similar brain activity when compared to the experience of aggressive thoughts and actions.

Despite the previous findings, several studies have found no relationship between violent video games and aggression (e.g., Przybylski et al., 2014; Toniutti et al., 2013). For example, in a longitudinal experiment, Kuhn and colleagues found no evidence that repeated exposure to a violent video game had any impact on aggressive behavior (Kühn et al., 2019). One issue that appears to emerge is that preregistered studies, wherein scholars post their analyses plans in advance so as to reduce questionable researcher practices, are particularly unlikely to find significant results (e.g., Hilgard et al., 2019; McCarthy et al., 2016). As such, more preregistered studies in this realm would be welcome.

Although individual studies may naturally differ in results, it is possible that meta-analyses may provide some illumination regarding larger trends in the field. However, there are disagreements in the conclusions of meta-analyses here too. For instance, Anderson and colleagues (2010) concluded that there are meaningful relationships between violent game playing and aggression in players. However, a reanalysis of this data concluded that effects, particularly for experimental studies, were largely driven by publication bias (Hilgard et al., 2017). Likewise, Prescott et al. (2018) found that there are very, very small longitudinal relationships between violent gameplay and later aggression. However, in reanalyzing this data, Drummond et al. (2020) concluded the effect sizes $(r=0.06)$ were trivial and driven by methodological noise, not true effects. Best practice studies demonstrated effect sizes that were no different from zero.

\section{The Current Study}

The purpose of this study was to determine whether violent content or acute stress plays a greater role in aggressiveness towards a cooperative partner while playing a video game. This study not only focused on investigating the role of stress in aggression but also its influence under varying conditions. The hypotheses being tested are as follows:

H1: Participants when exposed to acute stress will demonstrate greater aggressiveness toward an incompetent partner than a competent partner.

H2: Acute stress will play a greater role in causing aggressiveness towards an incompetent partner than chronic stress.

H3: Participants when exposed to stress and a violent video game will depict greater aggression toward an incompetent partner than those exposed to a nonviolent video game. 
We note that these hypotheses are expressed as main effects. However, it is possible that stress may moderate any relationship between violent game play and aggression (Shao \& Wang, 2019). As such, we will also be alert for interaction effects between the independent variables.

\section{Method}

Data Availability Statement A preregistration of this study can be found at https:// aspredicted.org/37ie4.pdf. Original data files can be found at https://osf.io/8w7mz/.

Conflict of Interest The authors declare no competing interests.

Informed Consent Informed consent was obtained from all individual participants included in the study.

\section{Participants}

This study involved 73 participants who were compensated with two extra credit points for psychology courses registered in the undergraduate research portal. About twenty percent of the participants were male $(20.5 \%, n=15)$, while the others were female $(79.5 \%, n=58)$. Participants' age ranged from 18 to 50 years old $(M=20.55$, $S D=4.98)$. Frequencies were also gathered for participants in each condition. In the stress condition, there were 37 participants $(50.7 \%)$ in the acute stress condition and $49.3 \%$ in the control condition $(n=36)$. For the video game condition, about half of the participants were in the violent condition $(52.1 \%, n=38)$ while 35 were in the nonviolent condition (47.9\%). Additionally, the incompetent confederate condition included 38 participants $(52.1 \%)$ while the competent condition consisted of $47.9 \%$ participants $(n=35)$. For a further two participants, a technical glitch resulted in the loss of their aggression data. In our preregistration document, we had planned to reach 100 participants. However, due to COVID-19 shutting down in-person research and the graduation of the lead author, participant recruitment was discontinued. This was done prior to examining any data.

\section{Measures}

State-Trait Anxiety Inventory (STAI) The State-Trait Anxiety Inventory (STAI) is a self-report anxiety measure (Spielberger et al., 1983). The inventory consists of a form Y-1, which measures current anxiety levels, and Y-2, which measures anxiety levels for the past 6 months. This measure contains 40 items, 20 state anxiety and 20 trait anxiety. For our purposes, we administered the Y2 trait form. Questions range from "I feel upset" to "I feel that difficulties are piling up so that I cannot overcome them." These questions were rated on a 4-point Likert scale ranging from almost never to almost always. For the trait anxiety form with the current sample, the coefficient alpha was 0.90 . The range of scores was from 22 to 70 from a possible range 
of $20-80$. The mean score was 71.49 with a standard deviation of 10.63 indicating good variability in scores.

Crossword Puzzle Participants took a simple crossword puzzle based on school classes, to act as a distractor task (see Appendix).

Stress Conditions In this study, participants were randomly assigned to one of two stress conditions: The Paced Auditory Serial Addition Task (PASAT) or a control math quiz (see Appendix). The PASAT was originally used as a means of assessing short-term memory loss; however, for this study, it was used as a measure of inducing stress in participants. In this study, the computerized version (PASAT-C) of the PASAT was used (Lejuez et al., 2003). Participants mentally calculated addition problems during a set time frame. The PASAT requires sustained and specific attention. Participants are given simple math problems, but these are done with intervening numbers that are distractors, thus setting up high frustration. Participants must add each number to the number before it, keying in the response. However, each response serves as a distractor. For instance, a respondent may be given the numbers, in sequence, 3, 9, and 5. For the first two numbers, they key in the correct response "12"; however, then, they are supposed to add 5 to the 9 . Yet, having " 12 " in working memory sets up a distractor and increases frustration, particularly as the task speeds up. Previous evidence has supported that the PASAT is effective in inducing stress, including for college students specifically (Holdwick, \& Wingenfeld, 1999; Starcke et al., 2016). Participants not assigned to the PASAT took a 10 -min math quiz meant to represent the no-stress condition. This quiz was not difficult. Examples of questions are finding the sum of 2 plus 2 and what is 5 multiplied by 5 .

Video Game Conditions For this study, participants played either a "violent" or "non-violent" video game on the XBOX One. Participants assigned to the violent game condition played Battlefront. Battlefront is an action-packed video game wherein the player was in control of a Star Wars character who wields a lightsaber or gun throughout gameplay. Those assigned to the "non-violent" condition played portal 2. Portal 2 is an action-adventure game rated E10+. This is a strategy video game wherein the player masqueraded as a first-person shooter wielding a portal gun which creates shortcuts throughout the game and does not cause harm to another character. Thus, the games are similar in gameplay and the use of a gun, but without violence of any kind in the portal 2 game.

Aggressive Behavior This study utilized a modified version of the Taylor competitive reaction time task (TCRTT). This is a newer version utilizing noise blasts instead of the electric shocks, used in the original study (Taylor, 1967). In Taylor's version, the participant had electrodes hooked up to their bodies and to convince the participant that they were playing against an individual they distributed a shock when the participant lost. He also had the wins and losses predetermined wherein the participant lost fifty percent of the trials. In this version, participants were asked 
to set the level and duration of a white noise blast acting as a punishment for their previous confederate partner. This game lasts for a total of $15 \mathrm{~min}$ and has 25 different trials. In the task, the wins and losses are preset as participants are not playing against a human opponent. The blast is not harmful and causes no discomfort; it is just annoying. As per the standardization suggested by Ferguson et al. (2008), aggressive behavior was measured as the averaged of the 25 intensity scores.

\section{Procedure}

Data collection began following Institutional Review Board approval. Participants who signed up under the undergraduate research portal were told that the researcher was conducting a study on the effect of several tests on an individual's ability to play games. To participate in this study, participants were asked to sign an informed consent form and to complete the STAI - Y2. Upon completion, they were asked to take a simple crossword puzzle, a distractor task to reduce hypothesis guessing. Participants were then randomly assigned to a stress condition, wherein they either took the PASAT-C or take a simple math quiz, both of which lasted $10 \mathrm{~min}$. After completing the stress condition activity, participants were then randomly assigned to a video game condition, wherein they either played Battlefront, a "violent" video game, or portal 2, a "non-violent" video game. Furthermore, they were randomly assigned to play the game for $30 \mathrm{~min}$ with either a competent or incompetent confederate partner. Once they entered the room, they were given instructions on how to operate an XBOX One controller and how to play the game they are assigned. After the game play, participants engaged with the TCRTT task. Participants were asked to play a reaction time game which lasted around $15 \mathrm{~min}$. For this game, they were informed that they were playing against their previous confederate, who is now in a separate room, and was competing against them on a separate computer. They were told that for every trial they can set the intensity and duration of white noise for their confederate as a form of punishment. Participants were also informed that they would hear the noise if they lost, while their partners would hear the noise if they won. They were told that they can set the noise level from 0 to 10 and that they can set the blast from 0 to $5 \mathrm{~s}$. Post gameplay, participants were debriefed, and the true nature of the study was revealed; all hypotheses were discussed, and they were asked if they held any suspicion of the topic's true hypotheses.

\section{Research Design}

To assess the effects of stress and video games on aggression, this study utilized a $2 \times 2 \times 2$ (stress $\times$ game condition $\times$ competence) factorial analysis of variance. The dependent variable was the noise blast intensity (aggression). To assure that randomization worked, and conditions were not confounded by demographic factors, chi-square analyses were run for gender, and bivariate correlations for age with a group assignment. No outcomes were statistically significant, suggesting randomization was effective. As such, age and gender will not be included as covariates. 


\section{Results}

\section{Screening, Cleaning, and Descriptive Statistics}

Once data collection was completed, data were entered and analyzed using IBM SPSS Version 26. A total of 73 participants partook in the study and received credit for their participation. Demographic data for the participants are presented in Table 1.

Descriptive statistics data are presented in Table 2.

\section{Hypotheses Tests}

A factorial ANOVA was conducted to evaluate the study hypotheses (Table 3). Firstly, there was no significant effect for the stress condition on the intensity setting by participants, $F(1,65)=0.04, p=0.85, \eta_{p}^{2}=0.001$. Similarly, there was also no significant effect for the video game condition on the intensity level setting, $F\left(1,65=0.09, p=0.77, \eta_{p}^{2}=0.001\right.$. The confederate condition also did not have a significant effect with the intensity setting, $F(1,65)=0.05, p=0.828, \eta_{p}^{2}=$ 0.001 . Hypothesis one stated that participants when exposed to acute stress will depict greater aggressiveness toward an incompetent partner than a competent partner. There was no significant finding for this hypothesis, as the stress condition and the confederate condition showed no significant interaction with the intensity level settings, $F(1,65)=2.05, p=0.16, \eta_{p}^{2}=0.03$. Related to hypothesis 3 , there was no significant impact of video game condition and stress condition $F(1,65)=0.002, p=0.97, \eta_{p}^{2}=0.03$. As such, hypothesis 3 was not supported.

Table 1 Demographic characteristics of study participants

\begin{tabular}{ll}
\hline Variable & Participants \\
\hline$N$ & 73 \\
Age $(M, S D)$ & $20.55,4.98$ \\
Gender $(N, \%)$ & \\
Male & $15(20.5)$ \\
Female & $58(78.5)$ \\
Stress condition $(N, \%)$ & \\
Acute stress & $37(50.7)$ \\
Chronic Stress & $36(49.3)$ \\
Video game condition $(N, \%)$ & \\
Violent & $38(52.1)$ \\
Non-violent & $35(47.9)$ \\
Competency condition $(N, \%)$ & \\
Incompetent & $38(52.1)$ \\
Competent & $35(47.9)$ \\
\hline
\end{tabular}


Table 2 Results of descriptive analyses for IVs with DV

\begin{tabular}{|c|c|c|c|c|c|}
\hline Stress condition & Video game condition & Confederate condition & $M$ & $S D$ & $N$ \\
\hline \multirow[t]{6}{*}{ High stress } & \multirow[t]{2}{*}{ Violent } & Incompetent & 4.66 & 2.29 & 10 \\
\hline & & Competent & 4.63 & 2.35 & 9 \\
\hline & \multirow[t]{2}{*}{ Non-violent } & Incompetent & 4.00 & 1.88 & 9 \\
\hline & & Competent & 5.52 & 1.76 & 9 \\
\hline & \multirow[t]{2}{*}{ Total } & Incompetent & 4.35 & 2.08 & 19 \\
\hline & & Competent & 5.08 & 2.07 & 18 \\
\hline \multirow[t]{6}{*}{ Low stress } & \multirow[t]{2}{*}{ Violent } & Incompetent & 4.92 & 2.14 & 10 \\
\hline & & Competent & 4.16 & 1.76 & 9 \\
\hline & \multirow[t]{2}{*}{ Non-violent } & Incompetent & 4.86 & 1.42 & 9 \\
\hline & & Competent & 4.53 & 1.44 & 8 \\
\hline & \multirow[t]{2}{*}{ Total } & Incompetent & 4.89 & 1.78 & 19 \\
\hline & & Competent & 4.33 & 1.58 & 17 \\
\hline \multirow[t]{6}{*}{ Total } & \multirow[t]{2}{*}{ Violent } & Incompetent & 4.79 & 2.16 & 20 \\
\hline & & Competent & 4.39 & 2.03 & 18 \\
\hline & \multirow[t]{2}{*}{ Non-violent } & Incompetent & 4.43 & 1.68 & 18 \\
\hline & & Competent & 5.05 & 1.65 & 17 \\
\hline & \multirow[t]{2}{*}{ Total } & Incompetent & 4.62 & 1.93 & 38 \\
\hline & & Competent & 4.71 & 1.86 & 35 \\
\hline
\end{tabular}

Dependent variable: INSMean

Table 3 Results of factorial ANOVA for study variables on the dependent variable

\begin{tabular}{llll}
\hline Independent variable or interaction & $F$ & $p$ & $\eta_{p}^{2}$ \\
\hline Stress condition & 0.036 & .85 & .001 \\
Video game condition & 0.088 & .77 & .001 \\
Confederate condition & 0.048 & .83 & .001 \\
Stress condition $\times$ Video game condition & 0.002 & .97 & .000 \\
Stress condition $\times$ confederate condition & 2.052 & .16 & .031 \\
Video game condition $\times$ confederate condition & 1.211 & .28 & .018 \\
Stress condition $\times$ video game condition $\times$ con- & 0.400 & .53 & .006 \\
$\quad$ federate condition & & & \\
\hline
\end{tabular}

Dependent variable: INSMean

\section{Exploratory Analysis (H2)}

We also examined the issue of whether acute stress played a greater role in causing aggressiveness towards an incompetent partner than chronic stress. For this, we conducted an OLS regression with pairwise deletion. Gender, STAI score (chronic stress), and stress condition (acute stress) were included as predictors. Although this was our $\mathrm{H} 2$ we note, as an oversight, we had not included the specifics of this analysis in our preregistration; thus, it should be considered exploratory. Ultimately, the regression model was non-significant $F(3,69)=0.017, p=0.997$. 
Given null results can be difficult to interpret all three major contrasts (game condition, stress condition, and confederate competence) were reassessed using Bayesian contrasts. In each case, Bayes factors indicated support for the null for game condition $(\mathrm{BF}=5.401)$, stress condition $(\mathrm{BF}=5.550)$, and confederate competence $(\mathrm{BF}=5.502)$. Thus, we are confident in interpreting results as supportive of the null.

\section{Discussion}

The purpose of this study was to determine whether stress or violent content played a role in aggressiveness towards a cooperative partner who is incompetent when playing a video game. Although there have been several studies on the influence of stress on aggression, there is still a lack of experimental studies discussing the influence of the different types of stress on aggression especially towards another individual, and whether that individual's actions play a role in increasing aggression. Moreover, this study attempted to examine the role of video games in aggression and whether they also influenced aggression towards another individual as this is important to several current concerns.

After conducting this study, there was no support for any of the study hypotheses. The results showed that the stress condition, whether it be acute (as primed by the PASAT) or chronic (as measured by the STAI), had no effect on the intensity setting participants gave their confederate partner. This finding illustrated that despite the participants' exposure to stress, there was no significant impact on their aggression, which means that the form of stress the individual is experiencing does not particularly influence their decision at least in this context. Furthermore, the stress condition and confederate competence condition in interaction had no significant impact on the aggression exhibited towards participants.

The video game condition also had no effect on the intensity of the noise burst participants administered to the confederate. The findings of this study were similar to those of previously mentioned studies (e.g., Hilgard et al., 2019; McCarthy et al., 2016). There was no significant difference in intensity level setting between participants in the violent or nonviolent group, thus illustrating that the type of video game experienced had no real effect on aggression towards another individual. Additionally, the confederate competence condition, interacting with the video game condition also had no significant effect on aggression towards the confederate, thus signifying that confederate competency and an individual's ability to further frustrate someone may not necessarily impact or increase their aggression towards them in this context. The absence of interaction effects was unable to support that stress may moderate links between violent games and aggressive behavior. Of course, it is possible that other moderators may function in other ways, but that was not evident from the current data.

Participants were also assigned to varying confederate conditions which exhibited two levels of competency, incompetent or competent, and these conditions had no significant effect on aggression. In studies like Hinsberger et al.'s (2016), it was found that participants administered shocks when individuals did not complete the judgment tasks correctly. This study's findings differ greatly and exhibit that 
competency itself does not necessarily impact aggression and other factors could play a role in the intensity level setting. For example, several participants described being competitive and disliked losing, while others exhibited far more passive feelings towards their confederate. As such, many participants may not have worried greatly about the competence of the confederate.

\section{Limitations}

As with all studies, ours has limitations. Our use of the STAI, in capturing anxiety, may have only considered a portion of chronic stress. It is possible that this measure may tap into personality characteristics as much as it does chronic stress. However, prior studies have used the STAI as a measure of chronic stress (e.g., al Abdi et al., 2018; Valsamakis et al., 2020). Our sample size was not as large as we had initially intended which naturally reduces the power of our analyses. Furthermore, generalizations from experiments on aggression that take place in the lab to real-life aggression should be done only with great care.

\section{Conclusions}

Future studies should look at other factors that may also influence aggression, especially when playing a game. For instance, competition would be a great variable to observe, especially as the aggression measure was viewed as a form of competitive task. The current study is a small one and, as noted, data collection was interrupted by COVID-19. However, in combination with other studies, it appears that violent content in video games has little impact on aggressive behavior, even in combination with acute stress. 


\section{Appendix 1}

\section{Puzzle distractor and math quiz}

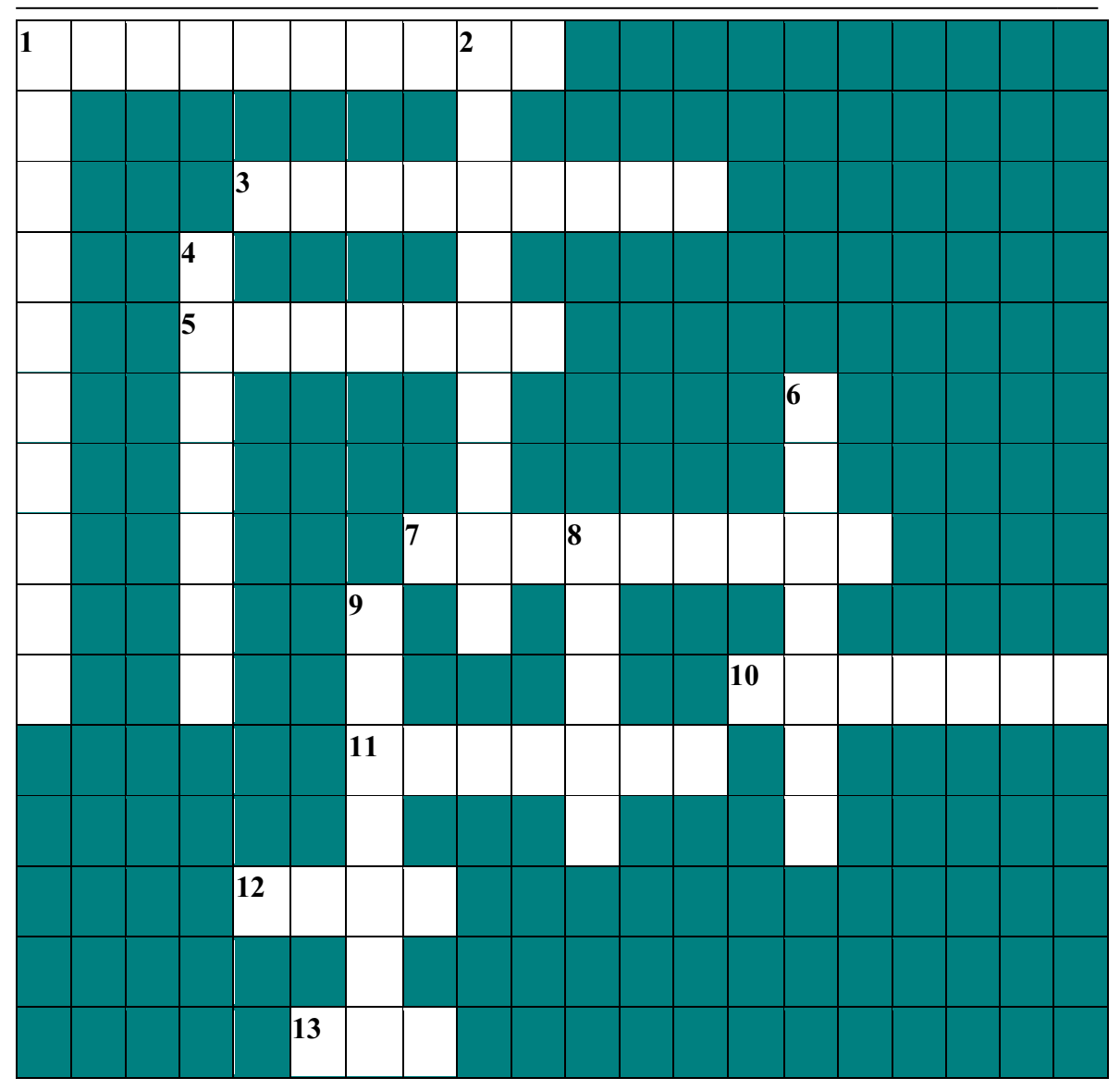

\section{Appendix 2}

\section{Crossword puzzle}

Please complete this crossword puzzle to the best of your ability. 


\section{Across:}

\section{Down:}

1. The study of the human mind.

1. The study of ideas and belief

3. In this class, you will learn about money and businesses. systems.

2. You will study a lot of maps in this

5. In this class, you might learn about ancient kings and queens. class.

4. In this class, you might learn about gravity or momentum.

7. The study of elements and chemicals.

6. To most American students,

10. The study of living things.

11. In this class, you might write an essay or read a play by Shakespeare.

12. You might need a calculator in this class.

13. Paint, brushes, and clay are all part of this class. French, Chinese, or Spanish are what languages.

8. In this class, you might sing or play an instrument.

9. This class teaches the dramatic arts.

Please answer each question to the best of your ability. You will have $10 \mathrm{~min}$. 


\begin{tabular}{lll}
\hline $1.40+5$ & $2.24 \times 8$ & $3.16 \div 4$ \\
4. $26 \times 3$ & $5.8+10$ & $6.15-9$ \\
7. $38 \div 2$ & $8.9+4$ & $9.18 \times 7$ \\
$10.3 \times 7$ & $11.60 \div 10$ & $12.12-7$ \\
$13.0+15$ & $14.13-8$ & $15.426-211$ \\
$16.10 \times 8$ & $17.13+6$ & $18.64 \div 4$ \\
$19.17-19$ & $20.43 \times 5$ & $21.19+5$ \\
$22.4+2$ & $23.843-720$ & $24.251-183$ \\
$25.40+51$ & $26.25 \div 4$ & $27.12+9$ \\
$28.8+5$ & $29.340-201$ & $30.72 \div 8$ \\
$31.29-20$ & $32.40 \times 7$ & $33.474-74$ \\
$34.7 \times 9$ & $35.7+16$ & $36.129+52$ \\
$37.0 \times 0$ & $38.652-3$ & $39.224+4$ \\
$40.6 \times 2$ & $41.63 \div 9$ & $42.91 \times 6$ \\
$43.1+107$ & $44.385+10$ & $45.303+19$ \\
$46.4 \times 3$ & $47.720+7$ & $48.436-410$ \\
$49.12 \times 12$ & $50.38 \times 2$ & $51.84 \div 7$ \\
$52.19-13$ & $53.385+3$ & $54.700-150$ \\
$55.22-15$ & $56.923-21$ & $57.183+6$ \\
$58.50 \div 5$ & $59.96 \div 8$ & $60.41 \times 9$ \\
& &
\end{tabular}

Data Availability A preregistration of this study can be found at https://aspredicted.org/37ie4.pdf. Original data files can be found at https://osf.io/ $8 \mathrm{w} 7 \mathrm{mz} /$.

\section{Declarations}

Informed Consent Informed consent was obtained from all individual participants included in the study.

Conflict of Interest The authors declare no competing interests. 


\section{References}

Al Abdi, R., Alhitary, A., Abdul Hay, E., \& Al-bashir, A. (2018). Objective detection of chronic stress using physiological parameters. Medical and Biological Engineering and Computing, 56, 2273-2286.

Anderson, C. A., Shibuya, A., Ihori, N., Swing, E. L., Bushman, B. J., Sakamoto, A., Rothstein, H. R., \& Saleem, M. (2010). Violent video game effects on aggression, empathy, and prosocial behavior in Eastern and Western countries: A meta-analytic review. Psychological Bulletin, 136(2), 151-173. https://doi.org/10.1037/a0018251

Augsburger, M., \& Maercker, A. (2020). Associations between trauma exposure, posttraumatic stress disorder, and aggression perpetrated by women. A meta-analysis. Clinical Psychology: Science and Practice, 27(1). https://doi.org/10.1037/h0101759

Clement, T. S., Parikh, V., Schrumpf, M., \& Fernald, R. D. (2005). Behavioral coping strategies in a cichlid fish: The role of social status and acute stress response in direct and displaced aggression. Hormones and Behavior, 47(3), 336-342. https://doi.org/10.1016/j.yhbeh.2004.11.014

Drummond, A., Sauer, J.D., \& Ferguson, C.J. (2020). Do longitudinal studies support long-term relationships between aggressive game play and youth aggressive behavior? A meta-analytic examination. Royal Society Open Science. https://doi.org/10.1098/rsos.200373

Ferguson, C. J., Rueda, S., Cruz, A., Ferguson, D., Fritz, S., \& Smith, S. (2008). Violent video games and aggression: Causal relationship or byproduct of family violence and intrinsic violence motivation? Criminal Justice and Behavior, 35, 311-332.

Ferguson, C. J., Copenhaver, A., \& Markey, P. (2020). Re-examining the findings of the APA's 2015 task force on violent media: A meta-analysis. Perspectives on Psychological Science, 15(6), 1423-1443.

Hilgard, J., Engelhardt, C. R., Rouder, J. N., Segert, I. L., \& Bartholow, B. D. (2019). Null effects of game violence, game difficulty, and 2D:4D digit ratio on aggressive behavior. Psychological Science, 30(4), 606-616. https://doi.org/10.1177/0956797619829688

Hilgard, J., Engelhardt, C. R., \& Rouder, J. N. (2017). Overstated evidence for short-term effects of violent games on affect and behavior: A reanalysis of Anderson et al. (2010). Psychological Bulletin, 143(7), 757-774. https://doi.org/10.1037/bul0000074

Hinsberger, M., Sommer, J., Kaminer, D., Holtzhausen, L., Weierstall, R., Seedat, S., \& Elbert, T. (2016). Perpetuating the cycle of violence in south african low-income communities: Attraction to violence in young men exposed to continuous threat. European Journal of Psychotraumatology, 7(1), 2909929109. https://doi.org/10.3402/ejpt.v7.29099

Holdwick, D. J., Jr., \& Wingenfeld, S. A. (1999). The subjective experience of PASAT testing: Does the PASAT induce negative mood? Archives of Clinical Neuropsychology, 14(3), 273-284. https:// doiorg.stetson.idm.oclc.org/10.1093/arclin/14.3.273

Kohl, C., Riccio, O., Grosse, J., Zanoletti, O., Fournier, C., Klampfl, S. M., \& Sandi, C. (2013). The interplay of conditional NCAM-knockout and chronic unpredictable stress leads to increased aggression in mice. Stress, 16(6), 647-654. https://doi.org/10.3109/10253890.2013.840824

Kühn, S., Kugler, D. T., Schmalen, K., Weichenberger, M., Witt, C., \& Gallinat, J. (2019). Does playing violent video games cause aggression? A longitudinal intervention study. Molecular Psychiatry, 24(8), 1220-1234. https://doi.org/10.1038/s41380-018-0031-7

Lejuez, C. W., Kahler, C. W., \& Brown, R. A. (2003). A modified computer version of the paced auditory serial addition task as a laboratory based stressor. The Behavior Therapist, 26(4), 290-293.

McCarthy, R. J., Coley, S. L., Wagner, M. F., Zengel, B., \& Basham, A. (2016). Does playing video games with violent content temporarily increase aggressive inclinations? A pre-registered experimental study. Journal of Experimental Social Psychology, 67, 13-19. https://doi.org/10.1016/j.jesp. 2015.10.009

Nosjean, A., Cressant, A., De Chaumont, F., Olivo-Marin, J., Chauveau, F., \& Granon, S. (2015). Acute stress in adulthood impoverishes social choices and triggers aggressiveness in preclinical models. Frontiers in Behavioral Neuroscience, 8, 447. https://doi.org/10.3389/fnbeh.2014.00447

Orth, U., \& Wieland, E. (2006). Anger, hostility, and posttraumatic stress disorder in trauma-exposed adults: A meta-analysis. Journal of Consulting and Clinical Psychology, 74(4), 698-706. https://doi. org/10.1037/0022-006X.74.4.698

Prescott, A. T., Sargent, J. D., \& Hull, J. G. (2018). Metaanalysis of the relationship between violent video game play and physical aggression over time. Proceedings of the National Academy of Sciences, 115(40), 9882-9888. https://doi.org/10.1073/pnas.1611617114 
Przybylski, A. K., Deci, E. L., Rigby, C. S., \& Ryan, R. M. (2014). Competence-impeding electronic games and players' aggressive feelings, thoughts, and behaviors. Journal of Personality and Social Psychology, 106(3), 441-457. https://doi.org/10.1037/a0034820

Rambo, C. L., Mocelin, R., Marcon, M., Villanova, D., Koakoski, G., de Abreu, M. S., Oliveira, T. A., Barcellos, L. J. G., Piato, A. L., \& Bonan, C. D. (2016). Gender differences in aggression and cortisol levels in zebrafish subjected to unpredictable chronic stress. Physiology \& Behavior, 171, 50-54. https://doi.org/10.1016/j.physbeh.2016.12.032

Shao, R., \& Wang, Y. (2019). The relation of violent video games to adolescent aggression: An examination of moderated mediation effect. Frontiers in Psychology, 10. https://doi.org/10.3389/fpsyg.2019. 00384

Spielberger, C. D., Gorsuch, R. L., Lushene, R., Vagg, P. R., \& Jacobs, G. A. (1983). Manual for the State-Trait Anxiety Inventory. Palo Alto, CA: Consulting Psychologists Press.

Starcke, K., Wiesen, C., Trotzke, P., \& Brand, M. (2016). Effects of acute laboratory stress on executive functions. Frontiers in Psychology, 7. https://doi.org/10.3389/fpsyg.2016.00461

Taylor, S. P. (1967). Aggressive behavior and physiological arousal as a function of provocation and the tendency to inhibit aggression. Journal of Personality, 35(2), 297. Retrieved from https://www.ncbi. nlm.nih.gov/pubmed/6059850. Accessed 1/20/22.

Toniutti, R., Born, M., \& Mathys, C. (2013). Les jeux vidéo violents augmentent-ils le biais d'attribution hostile chez des préadolescents? Retrieved from http://orbi.ulg.ac.be/handle/2268/149180. Accessed 1/20/22.

Valsamakis, G., Papatheodorou, D., Chalarakis, N., Manolikaki, M., Margeli, A., Papassotiriou, I., Barber, T. M., Kumar, S., Kalantaridou, S., \& Mastorakos, G. (2020). Maternal chronic stress correlates with serum levels of cortisol, glucose and c-peptide in the fetus, and maternal non chronic stress with fetal growth. Psychoneuroendocrinology, 114. https://doi.org/10.1016/j.psyneuen.2020.104591

Verona, E., \& Kilmer, A. (2007). Stress exposure and affective modulation of aggressive behavior in men and women. Journal of Abnormal Psychology, 116(2), 410-421. https://doi.org/10.1037/0021843X.116.2.410

Weber, R., Ritterfeld, U., \& Mathiak, K. (2006). Does playing violent video games induce aggression? empirical evidence of a functional magnetic resonance imaging study. Media Psychology, 8(1), 39-60. https://doi.org/10.1207/S1532785XMEP0801_4 